\title{
Criblage de variétés de tomate pour de la résistance au flétrissement bactérien causé par Ralstonia solanacearum au Togo
}

\author{
${ }^{1}$ Agnassim BANITO, ${ }^{1,2}$ Bitang BAMAZI, ${ }^{3}$ Essotina K. KPEMOUA, ${ }^{4}$ Rachidatou SIKIROU, \\ ${ }^{2}$ Pouwéréou TCHALLA, ${ }^{5}$ Mathews L. PARET \\ ${ }^{1}$ Ecole Supérieure d'Agronomie, Université de Lomé, B.P. 1515 Lomé, Togo \\ ${ }^{2}$ Institut de Conseil et d'Appui Technique, B.P. 65 Atakpamé, Togo \\ ${ }^{3}$ Institut Togolais de Recherche Agronomique, B.P. 1163 Lomé, Togo \\ ${ }^{4}$ Institut National des Recherches Agricoles du Bénin, 01 B.P 884 Cotonou, Bénin \\ ${ }^{5}$ University of Florida, North Florida Research and Education Center and Plant Pathology Department, 155 \\ Research Road, Quincy, FL 32351 \\ *Auteur-correspondant : Agnassim BANITO, PhD ; E-mail : bagnassim@ hotmail.com
}

Submitted on $3^{\text {rd }}$ August 2021. Published online at www.m.elewa.org/journals/ on $31^{\text {st }}$ October 2021

https://doi.org/10.35759/JABs.166.5

\section{RESUME}

Objectifs : Dans le cadre de la recherche de mesures de lutte efficace et durable contre le flétrissement bactérien causé par Ralstonia solanacearum $(R s)$, la présente étude s'est proposé d'évaluer les variétés de tomate pour leur résistance à la maladie.

Méthodologie et Résultats : Six variétés de tomate cultivées aux Togo ont été inoculées par une suspension de la souche de $R s$ isolée du site de CECO de la prefecture de Sotouboua (Togo). Les résultats ont montré de fortes incidences et sévérités du flétrissement bactérien sur les variétés testées. Cependant, des différences de comportement vis-à-vis de cette maladie ont été observées entre les variétés. En considérant l'indice de maladie après inoculation et l'analyse des closters, deux groupes de variétés ont été identifiés parmi les six testées : un groupe "Résistant" constitué de la seule variété Cobra et un groupe "Sensible" constitué des variétés Petomech, Tropimech, Padma, Roma et Platinum.

Conclusions et application des résultats : Cette étude a permis d'obtenir des données sur l'incidence et la sévérité du flétrissement bactérien cause par $R s$ et le comportement des variétés de tomate vis-à-vis de cette pathologie, et a permis aussi d'identifier les variétés Cobra, Roma et Platinum comme résistantes au flétrissement bactérien en conditions contrôlées. Ces résultats constituent une base de données importante pour des investigations futures en conditions de champs dans diverses zones agroécologiques du pays. Ces expérimentations permettront d'identifier des variétés résistantes pouvant être recommandées aux producteurs pour une gestion durable du flétrissement bactérien.

Mots clés : $R$. solancearum, flétrissement bactérien de la tomate, criblage, résistance. 


\section{ABSTRACT}

Objectives: In order to develop a control strategy against bacterial wilt caused by Ralstonia solanacearum $(R s)$, one of the most destructive diseases of tomato worldwide, the present study aimed to evaluate tomato varieties for resistance to $R s$.

Methodology and Results: Six tomato varieties cultivated in Togo were inoculated with Rs strain isolated from CECO site in the Prefecture of Sotouboua (Togo). The results revealed high incidence and severity occurring on these varieties. However, differences were observed among the tested varieties. The discrimination analysis based on the disease index after inoculation and the clusters analysis identified two groups of varieties: the resistant group composed of the varieties Cobra, Roma and Platinum and the susceptible group including the varieties Petomech, Tropimech, Padma.

Conclusions and application of findings: The results provided useful information in terms of incidence and severity of $R s$ wilt on tomato and the behaviour of varieties against the disease. The results allowed identifying three tomato varieties, Cobra, Roma and Platinum as resistant to bacterial wilt under controlled conditions. These results are useful for further experiments under field conditions in different agroecological zones to find out resistant tomato varieties for sustainable management of bacterial wilt caused by $R s$.

Keywords: $R$. solancearum, bacterial wilt of tomato, screening, resistance.

\section{INTRODUCTION}

La tomate (Solanum lycopersicum) est un légume-fruit majeur produit et consommé partout dans le monde (Boumendjel et al., 2012). Malheureusement, cette spéculation est sujette à de nombreuse contraintes biotiques qui peuvent réduire les rendements jusqu'à 90\% (Sikirou et al., 2011 et Ouédraogo, 2016). Parmi les maladies qui affectent la production de la tomate, figure le flétrissement bactérien de la tomate pouvant provoquer des pertes de rendement allant de 30\% à 90\% (Gumedzoe, 1993 ; Sikirou et al., 2011) voir 100\% (Sikirou et al., 2009 et Balamurugan et al., 2020). Des études récentes dans la préfecture de Sotouboua ont montré que cette spéculation est sujette au flétrissement bactérien avec une incidence de $10 \%$ et $50 \%$ (Kunwar et Bamazi et al., 2021). Au laboratoire, le test de verre d'eau et celui de flux latéral ou test sérologique immunostrip réalisés sur les échantillons ont été positif à $100 \%$ (Kunwar et Bamazi et al., 2021). Les colonies bactériennes sur le milieu SMSA ont été similaires à celles décrites par Sikirou et al. (2017); Sharma (2018) et Kunwar et Bamazi et al. (2021). Ces résultats ont été confirmés par des tests moléculaires à la PCR multiplex identifiant les isolats appartenant au sous-groupe de phylotype I, GMI 1000, Asiatica, Race 1 et biovar 3 (Kunwar et Bamazi et al., 2021). Le flétrissement bactérien de la tomate est largement répandu dans la Région Centrale du Togo d'après les investigations de Bamazi et al. (sous presse). La maladie vient d'être rapportée pour la première fois au Togo (Kunwar et Bamazi et al. 2021), et par conséquent aucune étude n'est à ce jour menée dans le cadre de la gestion de ce cancer de la tomate. La principale méthode de lutte contre le flétrissement bactérien de la tomate, comme pour la plupart des affections bactériennes des cultures, est l'utilisation des variétés résistantes (Sidikou et al., 2005 ; Fondio et al., 2013 et Ouédraogo, 2016). C'est dans cette optique de développer une stratégie de gestion efficace et durable contre le flétrissement bactérien de la tomate causé par $R$. solanacearum que la présente étude tire son importance et se propose d'évaluer quelques variétés de tomate pour leur résistance à cette affection. 


\section{MATERIEL ET METHODES}

Site expérimental : L'expérience a été conduite sous serre à la Station Expérimentale Agronomique de l'École Supérieure d'Agronomie de l'Université de Lomé. Cette serre est équipée d'un système d'aération, avec une température intérieure de 28 à $30^{\circ} \mathrm{C}$.

Matériel végétal : Six variétés certifiées de tomate cultivées dans la Région Centrale ont été testées : Petomech, Tropimeck, Platinum, Roma, Padma et Cobra. Ces variétés ont été obtenues auprès de la société de commercialisation de semences maraichères Togosemences, et sont largement cultivées dans la région centrale en milieu paysan. Une pépinière a été faite pour chaque variété. Des pots en plastique ont été remplis du terreau préalablement stérilisé à l'étuve à $80{ }^{\circ} \mathrm{C}$ pendant 72 heures, puis arrosés.

Dispositif expérimental : La souche de $R$. solanacearum utilisée est la T18 isolée à partir de plant flétri de tomate prélevé sur le site de CECO-AGRO dans la préfecture de Sotouboua. Cette souche s'est montrée plus virulente que trois autres provenant des préfectures de Tchamba, Tchaoudjo et Blitta. Le dispositif expérimental pour le criblage est celui en bloc complètement aléatoire à cinq.

Suspension bactérienne et inoculation des plants: L'inoculum à partir d'une culture bactérienne pure de 72 heures de la souche T18 de $R$. solanacearum sur l'agar nutritif, avec de l'eau distillée stérile pour obtenir une suspension bactérienne de $10^{8} \mathrm{CFU} / \mathrm{ml}$. Les jeunes plants de 21 jours après semis ont été inoculés avec la suspension bactérienne préparée, selon la technique d'inoculation proposée par Fondio et al. (2013) et Oussou et al. (2020). Elle consiste à tremper les racines de différente variété de tomate légèrement blessées à l'aide d'un scalpel dans $50 \mathrm{ml}$ de la suspension bactérienne pour chaque variété pendant 5 minutes. Les plants inoculés sont ensuite repiqués dans les pots. Puis $50 \mathrm{ml}$ de la suspension bactérienne a été versé au pied de chaque plant. Une deuxième inoculation à raison de $50 \mathrm{ml}$ de la suspension de $10^{8}$ $\mathrm{CFU} / \mathrm{ml}$ a été faite une semaine après la première. Les plants repiqués ont été arrosées après 24 heures d'inoculation, puis de façon régulière pour une humidité convenable au développement des plants.

Collecte des données : L'évaluation est faite par observations des parties aériennes des plantes à la détection de symptômes de flétrissement à intervalle de 5 jours. Ces observations ont permis d'évaluer aussi bien l'incidence que la sévérité du flétrissement des plants après inoculation.

L'incidence de la maladie a été évaluée en pourcentage du nombre de plants malades quel que soit le degré de symptômes suivant la formule :

$$
\mathrm{I}=(\mathrm{NPF} / \mathrm{NTP})^{*} 100
$$

$\mathrm{I}$ : incidence du flétrissement bactérien ;

NPF : nombre de plants flétris ;

NPT : nombre total de plants évalués.

Le comportement des variétés vis-à-vis du flétrissement bactérien après inoculation a été déterminé en estimant la sévérité de la maladie suivant l'échelle de cotation de 5 classes utilisée par Ciampi et al. (1980), He et al. (1983), Swanepoel (1990) et Sidikou Seyni et al. (2005) (Tableau 1). 
Tableau 1 : Échelle de cotation de 1 sévérité de maladie

\begin{tabular}{c|l|c|c}
\hline Classe & \% de maladie & Index $(\mathbf{C i})$ & Nombre de plants $(\mathbf{N i})$ \\
\hline 0 & 0 & 0 & $\mathrm{~N} 0$ \\
1 & $<5(1$ feuille flétrie) & 0,025 & $\mathrm{~N} 1$ \\
2 & 5 à 25 & 0,150 & $\mathrm{~N} 2$ \\
3 & 25 à 50 & 0,375 & $\mathrm{~N} 3$ \\
4 & 50 à 75 & 0,625 & $\mathrm{~N} 4$ \\
5 & 75 à 100 & 0,875 & $\mathrm{~N} 5$ \\
\hline
\end{tabular}

$\mathrm{Ci}=$ index ou coefficient correspondant à chaque classe ; $\mathrm{Ni}=$ nombre de plants avec le coefficient $\mathrm{Ci}$.

Cette cotation a permis de déterminer l'indice de la maladie (IMr) selon la formule suivante (Winstead et Kelman, 1952 ; Adamou, 2011 ; Oussou et al., 2020) :

$$
\mathrm{IMr}=\sum \mathrm{Ci} * \mathrm{Ni} / \sum \mathrm{Ni}
$$

Selon la valeur de de l'indice de maladie IMr, des classes de comportement des variétés ont été définies (Sidikou Seyni et al., 2005) :

$\mathrm{IMr}<0,2$ : comme variété résistante ; $0,2 \leq \mathrm{IMr} \leq 0,5:$ comme variété tolérante ; IMD > 0,5: comme variété sensible.

Analyse des données : Les données collectées ont été traitées avec le logiciel de calcul Excel.
L'analyse de la variance (ANOVA) des données relatives à l'incidence, la sévérité et l'indice de maladie du flétrissement bactérien après inoculation été faite à l'aide du logiciel Genstat Discovery Edition 4. Les moyennes des valeurs de ces différents paramètres ont été discriminées en utilisant test de Student Newman Keuls au seuil de 5\%. Le dendogramme a été construit par l'analyse de clusters à l'aide du logiciel SPSS Statistics Version 25 à partir des données de l'indice maladie (IMr) du flétrissement bactérien.

\section{RESULTATS}

Symptômes du flétrissement après feuilles (Figure 1a), puis devenant plus l'inoculation des plants : Les symptômes de flétrissement ont été observés de façon progressive, touchant d'abord les jeunes sévères, envahissant toute la plante (Figure 1b) pour les variétés sensibles.
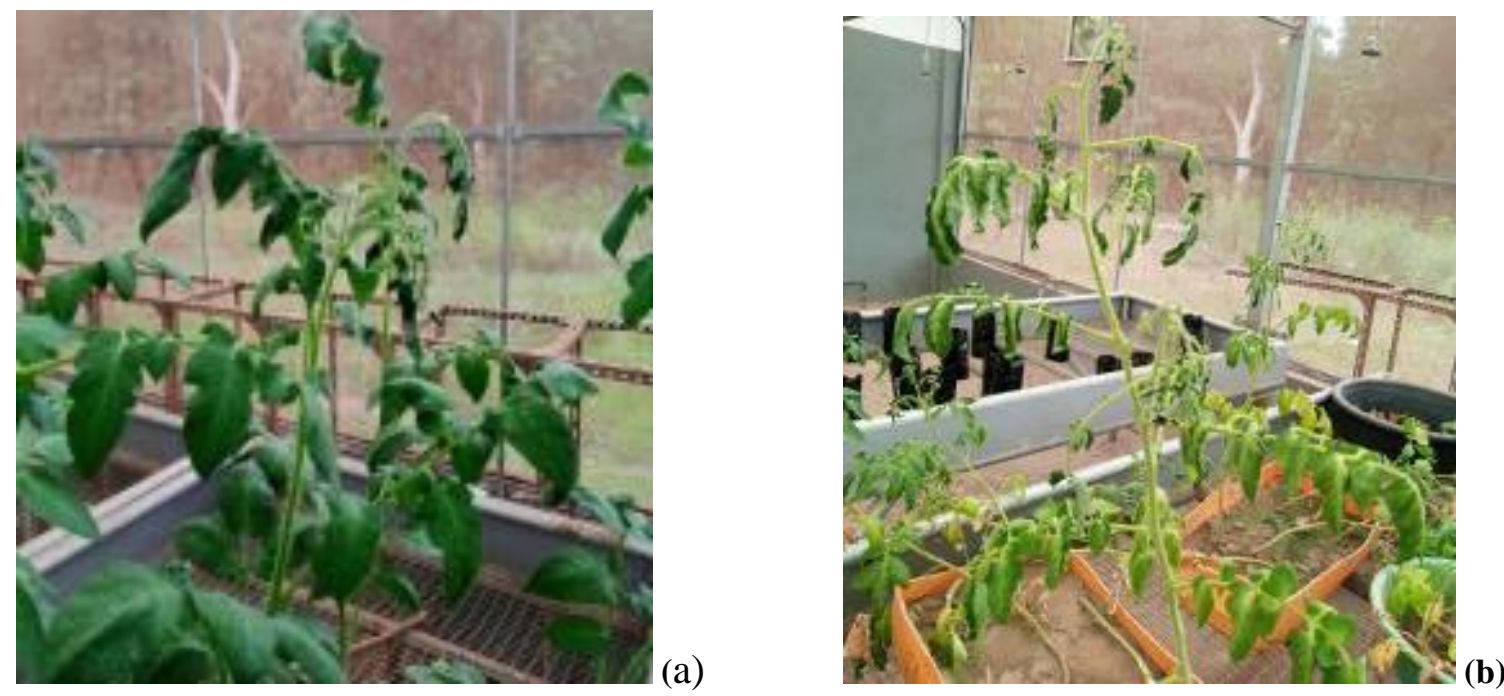

Figure 1 : Plants présentant des symptômes de flétrissement bactérien après inoculation 
a) début de flétrissement ; b) flétrissement avancé.

Incidence du flétrissement bactérien après inoculation : L'incidence $\mathrm{du}$ flétrissement bactérien des plants inoculés varie de 0 à $100 \%$. Les variétés Petomech, Tropimech et Padma ont un taux d'incidence de 40\% 30 jours après l'inoculation (JAI). Pour la variété Platinum, le flétrissement a commencé à 40 JAI avec une incidence de $40 \%$ qui a évolué jusqu'à $60 \%$ à $55 \mathrm{JAI}$, puis a régressé pour se stabiliser à $20 \%$ à partir de 70 JAI. La variété Roma a eu un maximum de $60 \%$ d'incidence, alors que Cobra n'a enregistré que 20 à $40 \%$ d'incidence entre 40 et 55 JAI, puis n'a plus développé la maladie (Figure 2). En considérant la moyenne de l'incidence sur l'ensemble des dates d'évaluation des symptômes, les variétés Petomech, Tropimech et Padma ont enregistré les incidences les plus élevées avec des taux dépassant les 70\%, significativement supérieurs à ceux des trois autres variétés testées $(\mathrm{P}<0,05)$. La variété Cobra a présenté le plus faible taux d'incidence avec une moyenne de 6,2\%, significativement inférieure $(\mathrm{P}<0,05)$ à celles des variétés Platinum et Roma qui ont enregistré un taux d'incidence de 29,2\% chacune (Tableau 2).

Tableau 2: Incidence moyenne du flétrissement bactérien sur les variétés de tomate après inoculation

\begin{tabular}{l|l}
\hline Variétés & Incidence Moyenne $(\%)$ \\
\hline Petomech & $76,9 \pm 21,36 \mathrm{a}$ \\
Tropimech & $83,2 \pm 22,87 \mathrm{a}$ \\
Platinum & $29,2 \pm 21 \mathrm{~b}$ \\
Roma & $29,2 \pm 21 \mathrm{~b}$ \\
Padma & $70,5 \pm 4,13 \mathrm{a}$ \\
Cobra & $6,2 \pm 12,61 \mathrm{c}$ \\
\hline Moyenne & $49,2 \pm 24,93$ \\
\hline
\end{tabular}

$\mathrm{LSD}=19,49 ; \mathrm{SE}=24,93 ; \mathrm{CV}=50,6 \%$; Les valeurs de la colonne portant la même lettre sont statistiquement identiques au seuil de $5 \%$.

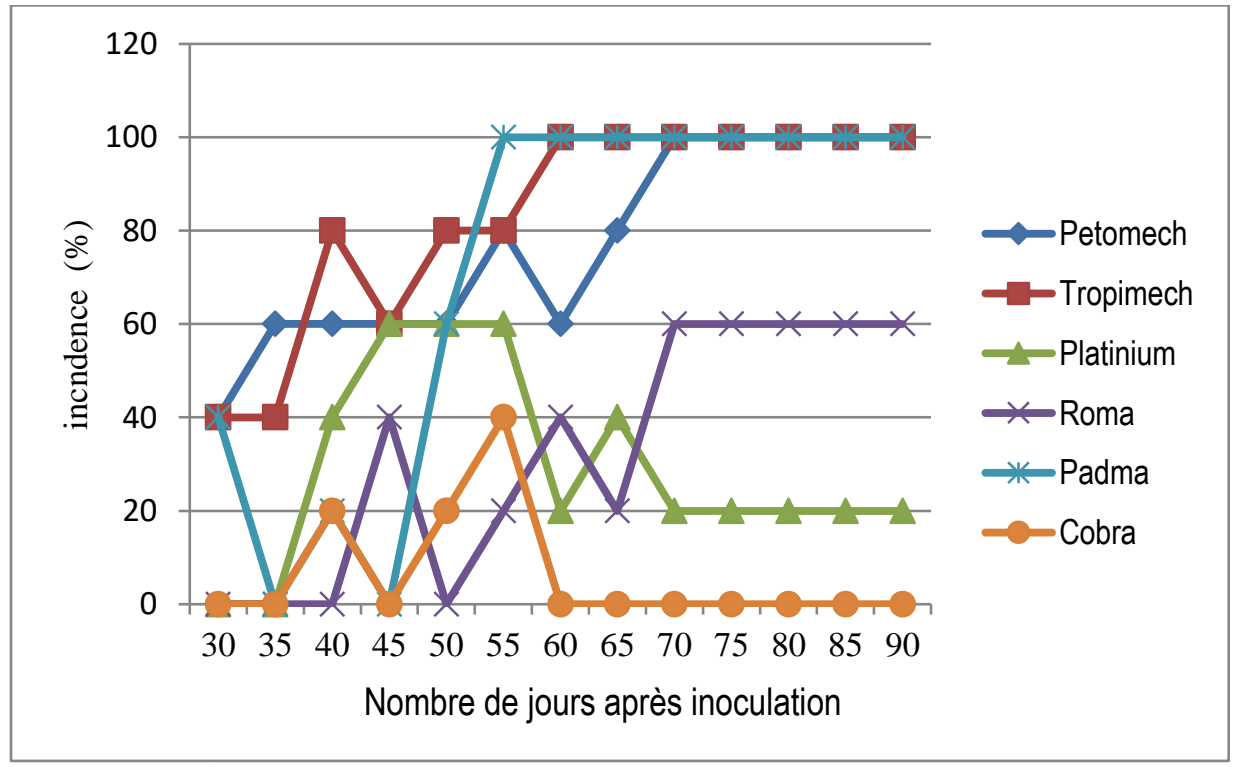

Figure 2 : Évolution de l'incidence du flétrissement bactérien sur les variétés de tomate après inoculation 
Sévérité du flétrissement bactérien sur les variétés de tomate après inoculation : $\mathrm{La}$ sévérité du flétrissement bactérien après inoculation a été de moins de $1 \%$ à $56,4 \%$ pour l'ensemble des variétés testées. Comme pour l'incidence, les variétés Petomech, Tropimech et Padma ont enregistré les sévérités les plus élevées avec des taux 50,9\% à 53,4\%, significativement supérieurs $(\mathrm{P}<0,05)$ à ceux des Platinum, Roma et Cobra, qui elles, ont enregistré des sévérités très faibles statistiquement identiques de $0,027 \%$ à $4,7 \%$ (Tableau3). Les données ont permis de regrouper les 6 variétés testées en deux classes de sévérités suivant l'échelle de cotation adoptée. Les variétés Petomech, Tropimech et Padma sévèrement attaquées sont rangées dans la classe 4, tandis que les variétés Platinum, Roma et Cobra qui ont enregistré des taux de sévérités inférieurs à $5 \%$ et sont rangées dans classe 1 (Tableau3).

Tableau 3: Sévérité du flétrissement bactérien sur les variétés de tomate après inoculation

\begin{tabular}{l|l|c}
\hline \multirow{2}{*}{ Variétés } & \multicolumn{2}{c}{ Sévérité } \\
\cline { 2 - 3 } & Taux (\%) & Classe \\
\hline Petomech & $50,9 \pm 14,9 \mathrm{a}$ & 4 \\
Tropimech & $53,7 \pm 11,7 \mathrm{a}$ & 4 \\
Platinum & $4,7 \pm 6,96 \mathrm{~b}$ & 1 \\
Roma & $1,5 \pm 3,44 \mathrm{~b}$ & 1 \\
Padma & $56,4 \pm 10,24 \mathrm{a}$ & 4 \\
Cobra & $0,027 \pm 0,91 \mathrm{~b}$ & 1 \\
\hline Moyenne & $27,9 \pm 9,35$ & \\
\hline
\end{tabular}

$\mathrm{LSD}=12,2 ; \mathrm{SE}=9,35 ; \mathrm{CV}=33,5 \%$

Corrélation l'incidence et la sévérité du flétrissement bactérien sur les variétés de tomate après inoculation: L'analyse de l'interaction entre l'incidence et la sévérité du flétrissement bactérien a révélé une corrélation entre ces deux paramètres pour toutes les variétés testées. En effet, l'analyse a indiqué une forte corrélation positive les deux paramètres avec des coefficients de corrélation allant de 0,857178 pour la variété Padma à 1 pour la variété Roma (Tableau4). Ceci indique que l'augmentation de l'incidence du flétrissement bactérien sur les plants de tomate entraîne une augmentation de la sévérité de cette pathologie chez ces plants.

Tableau 4 : Interaction entre l'incidence et la sévérité du flétrissement bactérien sur les variétés de tomate après inoculation

\begin{tabular}{l|l|l|l}
\hline Variétés & Incidence $\mathbf{( \% )}$ & Sévérité $\mathbf{( \% )}$ & Corrélation \\
\hline Petomech & $76,9 \pm 21,36 \mathrm{a}$ & $50,9 \pm 14,9 \mathrm{a}$ & 0,954656 \\
Tropimech & $83,2 \pm 22,87 \mathrm{a}$ & $53,7 \pm 11,7 \mathrm{a}$ & 0,962418 \\
Platinum & $29,2 \pm 21 \mathrm{~b}$ & $4,7 \pm 6,96 \mathrm{~b}$ & 0,95176 \\
Roma & $29,2 \pm 21 \mathrm{~b}$ & $1,5 \pm 3,44 \mathrm{~b}$ & 1 \\
Padma & $70,5 \pm 4,13 \mathrm{a}$ & $56,4 \pm 10,24 \mathrm{a}$ & 0,857178 \\
Cobra & $6,2 \pm 12,61 \mathrm{c}$ & $0,027 \pm 0,91 \mathrm{~b}$ & 0,993957 \\
\hline Moyenne & $49,2 \pm 24,93$ & $27,9 \pm 9,35$ & 0,982337 \\
\hline
\end{tabular}

Lsd=19,49; se=24,93; $\mathrm{cv}=50,6 \%$ (pour l'incidence) ; $1 \mathrm{sd}=12,2 ; \mathrm{se}=9,35 ; \mathrm{cv}=33,5 \%$ (pour la sévérité). Les valeurs d'une même colonne portant la même lettre sont statistiquement identiques au seuil de $5 \%$. 
Indice de maladie du flétrissement bactérien sur les variétés de tomate après inoculation : L'indice de maladie a varié de 0,03 pour la variété Cobra à 2,68 pour la variété Tropimech. Les variétés Platinum et Roma avec des taux d'incidence et de sévérités faibles par rapport aux variétés Petomech, Tropimech et Padma, ont enregistré des indices de maladies respectifs de 0,58 et de 0,65. Cependant, par rapport à l'échelle de classification adoptée, seule la variété Cobra a enregistré un indice de maladie inférieure à $0,2 \%$ et se retrouve comme seule variété résistante des 6 testées vis-à-vis du flétrissement bactérien causé par $R$. solanacearum. Les 5 autres variétés ont obtenu des indices de maladie supérieurs à 0,5 et sont donc toutes sensibles au flétrissement bactérien (Tableau 5). Comme pour la discrimination à base de l'indice de maladie $\mathrm{IMr}$, le dendogramme issu de l'analyse des clusters classe les six variétés en deux groupes. Sauf que pour l'analyse des clusters le groupe « Résistant » au flétrissement bactérien dû à $R$. solanacearum est constitué, en plus de la variété Cobra, des variétés Roma et Platinum, tandis que le groupe «Sensible » renferme les variétés Padma, Tropimech, Petomech (Figure $3)$.

Tableau 5: Indice de maladie du flétrissement bactérien sur les variétés de tomate après inoculation

\begin{tabular}{l|c|c}
\hline Variétés & IMr & Classe de résistance \\
\hline Petomech & 2,31 & $\mathrm{~S}$ \\
Tropimech & 2,68 & $\mathrm{~S}$ \\
Platinum & 0,58 & $\mathrm{~S}$ \\
Roma & 0,65 & $\mathrm{~S}$ \\
Padma & 2,62 & $\mathrm{~S}$ \\
Cobra & 0,03 & $\mathrm{R}$ \\
\hline
\end{tabular}

$\mathrm{IMr}=$ indice de maladie $; \mathrm{S}=$ sensible $; \mathrm{R}=$ résistante. 


\section{Dendrogramme utilisant la Distance complète}

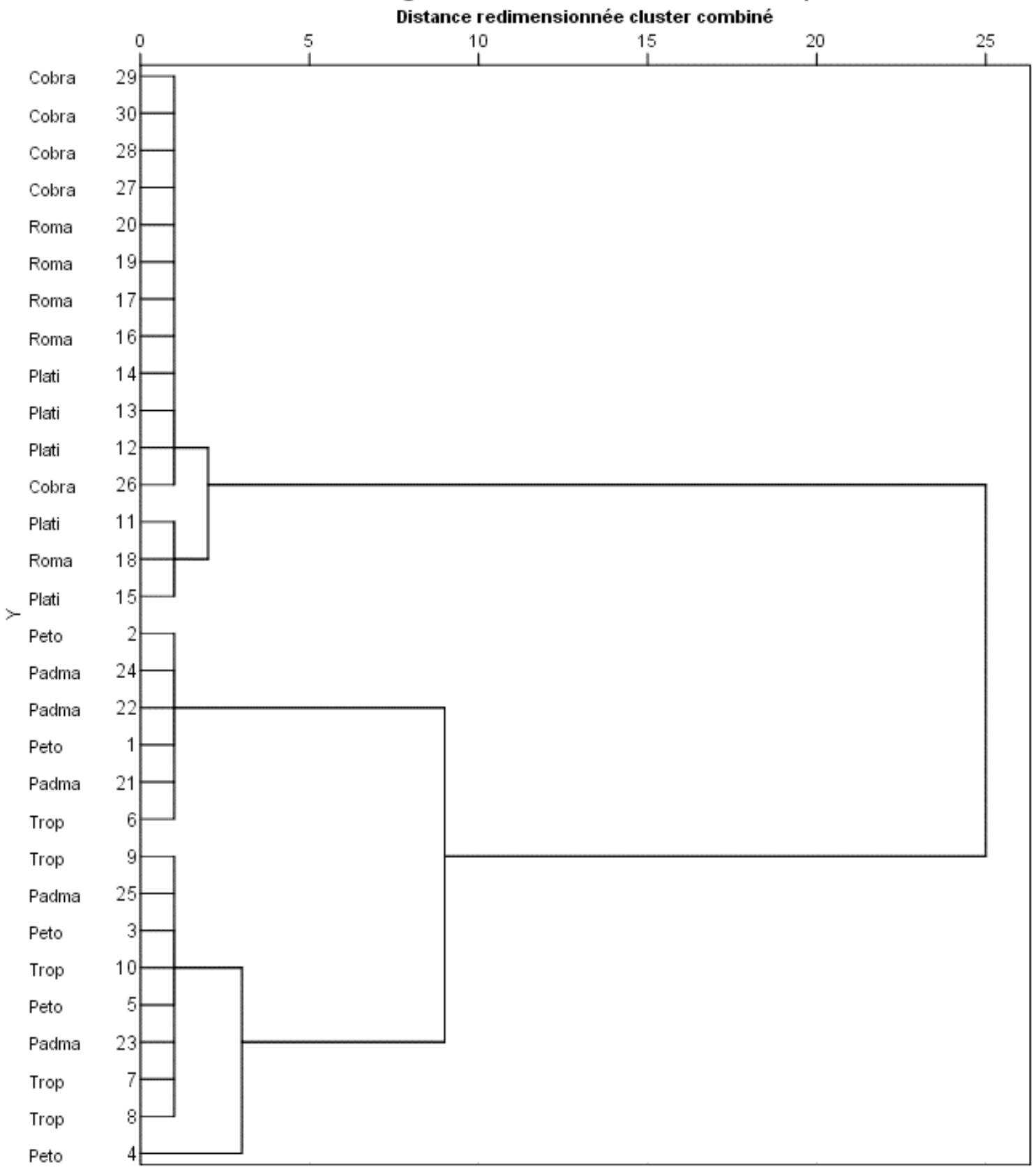

Figure 3 : Dendrogramme montrant 2 sous-groupes après le criblage.

Rs $=$ Ralstonia solanacerum $;$ Plati $=$ Platinum $;$ Trop $=$ Tropimech $;$ Peto $=$ Petomech.

\section{DISCUSSION}

Les symptômes obtenus lors du criblage sont similaires à ceux observés dans les champs de producteurs et caractéristiques du flétrissement bactérien causé par la bactérie $R$. solanacearum. A l'exception de la variété Cobra qui a présenté de faibles symptômes, les autres variétés ont été malades avec d'importants symptômes de flétrissement. Ces résultats ont montré une différence de comportement entre les variétés testées vis-àvis de cette pathologie, donc des niveaux de sensibilité différents. En Côte d'Ivoire, les 
travaux de N'guessan et al. (2019) ont révélé différents niveaux de sensibilité au flétrissement bactérien parmi des variétés de tomate, allant de hautement sensibles au plus résistantes. Parmi les variétés testées, Petomech et Tropimech sont les plus cultivées par les producteurs dans la Région Centrale. Malheureusement, ces deux variétés se trouvent être des plus sensibles parmi les variétés soumises au test de criblage. Ces résultats confirment les observations de Bamazi et al. (2019) lors des prospections, et les rapports de l'ICAT-Centrale (2020) faisant état de plaintes récurrentes des producteurs liées au flétrissement des plants de tomate dans leurs exploitations, engendrant des pertes de rendement. La variété Cobra a manifesté quelques faibles symptômes autour des 40 premiers jours après inoculation, mais ces symptômes ont disparu au cours des jours suivants; cela pourrait s'expliquer par la capacité de cette variété à vaincre la maladie, aptitude observable chez des variétés résistantes à une affection donnée, et donc la variété Cobra serait une candidate à la stratégie de gestion durable de cette pathologie. Blancard et al. (2020) rapporte que l'utilisation des variétés résistantes pour contrôler le flétrissement bactérien de la tomate causé par $R$. solanacearum est la solution la plus efficace, économique et respectueuse de l'environnement. L'incidence du flétrissement bactérien obtenu dans cette étude a varié de $6 \%$ à $80 \%$. De récents travaux ont montré que l'incidence du flétrissement bactérien dans la préfecture de Sotouboua, en 2018, variait entre $10 \%$ et $50 \%$ (Kunwar et Bamazi et al., 2021). Des taux d'incidence élevés de $76 \%$ à $100 \%$ ont été rapportés dans les Caraïbes par Deberdt et al (2013). Ces forts taux d'incidence pourraient engendrer d'énormes pertes de production de tomate aux producteurs. Fondio et al. (2010) ont rapporté des pertes significatives de rendement atteignant $80 \%$ à $100 \%$, faisant de du flétrissement bactérien de la tomate la bactériose la plus nuisible au monde compte tenu de sa distribution mondiale, sa large gamme d'hôtes concerné et de l'impact socio-économique (Kunwar et Bamazi et al., 2021). Dans la présente étude, les variétés les Tropimech, Petomech et Padma ont présenté des incidences de flétrissement significativement plus élevées que les variétés Platinum et Roma moyennement atteintes et la variété Cobra très faiblement affectée. La réaction variable des variétés de tomate en termes d'incidence du flétrissement bactérien a été rapporté (INRA, 2020). La sévérité du flétrissement bactérien après inoculation a été diversement exprimée par les variétés testées. Comme pour l'incidence, les variétés Petomech, Tropimech et Padma ont été significativement plus sévèrement affectées que les variétés Platinum, Roma et Cobra. Les résultats ont révélé que l'augmentation de l'incidence entraîne une augmentation de la sévérité du flétrissement bactérien. $\mathrm{La}$ discrimination basée sur l'indice de maladie a révélé que la variété Cobra a été la seule résistante au flétrissement bactérien après inoculation et les cinq autres variétés ont été sensibles. Cependant, selon le dendrogramme issu, les variétés Roma et Platinum se retrouvent ensemble dans le groupe «Résistant» avec la variété Cobra. Ces résultats sont confirment en partie ceux de Deberdt et al (2013) qui ont rapporté que la variété de tomate Cobra était résistante au flétrissement bactérien dans les Caraïbes. Le flétrissement bactérien est une maladie mondiale très dommageable. Les présents résultats montrent l'importance et la gravité de la maladie en termes d'incidence et de sévérité. Ces résultats en conditions contrôlées constituent une base scientifique solide pour des investigations ultérieures en conditions réelles en vue d'identifier des variétés résistantes pour une production durable de la tomate au Togo. 


\section{REFERENCES}

Adamou I, 2011. Caractérisation des douches locales de Ralstonia solanacearum (E. F. Smith) Yabuuchi et al. et évaluation du comportement de six variétés de pomme de terre (Solanum tuberosum L.) face à la souche Rs-09-76, au Niger. Thèse de doctorat à l'Université de Ouagadougou, $197 \mathrm{p}$.

Balamurugan A, Muthamilan M, Kamalakannan A, Shanthi A et Arumugam T, 2020. Characterization of Ralstonia solanacearum Causing Bacterial Wilt Disease of Tomato in Coimbatore District of Tamil Nadu, India. Int. J. Curr. Microbiol. App. Sci, 9(2) : 3010-3016.

Bamazi B, Banito A, Ayisah KD, Sikirou R, Paret LM, Tchalla $\mathrm{P}$, Kamde $\mathrm{K}$ et Nimblika T, 2019. Incidence et sévérité des flétrissements bactérien et fongique de la tomate au Togo : cas de la Région Centrale. L'Université africaine au cour de la création des valeurs : l'académique et la recherche en questions. Colloque Scientifique International de l'Université de Kara, 23-27 septembre 2019, p. 122.

Blancard D, Marchoux G, Laterrot $\mathrm{H}$ et Candresse T, 2020. Les maladies de la tomate. Identifier, connaître, maîtriser. INRA- Techniques et Pratiques. https://www.quae.com/produit/844/ 9782759213627/les-maladies-de-latomate

Boumendjel M, Houhamdi M, Samar MF, Sabeg H, Boutebba A et Soltane M, 2012. Effet des traitements thermiques d'appertisation sur la qualité biochimique, nutritionnelle et technologique du simple, double et triple concentre de tomate. Sciences et Technologie. C, Biotechnologies, (36) : 51-59.

Ciampi L, Sequeira L and French ER, 1980. Latent infection of potato tubers by
Pseudomonas solanacearum. Am. Potato J., 57 : 377-386.

Deberdt P, Pinon GM, Coranson BR, Etienne L et Fernandes P, 2013. Les nouvelles variétés de tomate sont-elles tolérantes au flétrissement bactérien? In : Conférence finale de projet DEVAG Réseau caribéen pour le développement des systèmes horticoles agroécologiques. CIRAD. SI : $1 \mathrm{p}$.

Fondio L, Djidji AH, N'gbesso MFDP et Ochou OG, 2010. Évaluation des variétés de tomate et piment pour la tolérance au flétrissement bactérien, et multiplication des semences de piment. Rapport d'activités, Convention CNRA/AVRDC, CNRA, Bouaké, Côte d'Ivoire, $31 \mathrm{p}$.

Fondio L, Djidji HA, N'Gbesso F et Kone D, 2013. Évaluation de neuf variétés de tomate (Solanum lycopersicum L.) par rapport au flétrissement bactérien et à la productivité dans le Sud de la Côte d'Ivoire. International Journal of Biological and Chemical Sciences, 7(3): 1078-1086.

Gumedzoe MYD, 1993. Les principaux virus du niébé au Togo. Cahier Agriculture, $2: 352-55$.

He LY, Sequeira L and Kelman A, 1983. Characteristics of strains of Pseudomonas solanacearum from China. Plant Disease, 67 : 1357-1361.

ICAT-Centrale, 2020. Rapport annuel d'activités. Exercice agricole 2020, Togo.

INRA, 2020. Alimentation, agriculture, environnement : une prospective pour la recherche. Rapport Inra, Paris.

Kunwar S, Bamazi B, Banito A, Carter M, Weinstein S, Steidl O, Hayes MM, Allen C, Paret M, 2021. First Report of Bacterial Wilt Disease of Tomato, Pepper and Gboma Caused by Ralstonia solanacearum Species 
Complex in Togo. Plant Disease, 105(2) : 484

N'guessan BR, Adingra KBC, Komena BK, Ipou IJ, 2019. Les Facteurs de Risques de la Modernisation de l'agriculture à Base de Pesticides dans la SousPrefecture de Tapeguia. European Scientific Journal, 15(15) : 378-400.

Ouédraogo WK, 2016. Caractérisation des isolats de Ralstonia solanacearum (EF Smith) Yabuuchi et al., agent causal du flétrissement bactérien dans les périmètres maraichers du Houet, du Knédougou et du Passore et recherche de variétés résistantes à la maladies. Mémoire d'ingénieur du développement rural, Université Polytechnique de Bobo-Dioulasso, 66 p.

Oussou GF, Sikirou R, Afoha SA, Dossoumou ME, Boukari SA, Komlan FA et Zocli B, 2020. Resistance assessment of tomato (Solanum lycopersicum L.) and gboma (Solanum macrocarpon L.) Cultivars against bacterial wilt caused by Ralstonia solanacearum in Benin. Pakistan Journal of Phytopathology, 32(2) : 241-249.

Sharma DK, 2018. Morphological and biochemical characterization of Ralstonia solanacearum (smith) in Brinjal (Solanum melongena L.) in Rajasthan (India). Adv. Plants Agric Res., 8(3) : 284-288.

Sidikou RDS, Sihachakr D, Le Roux AC, Servaes A, Ambroise A, Jouan B, Ellisseche D et Ducreux G, 2005. Etude de la résistance in vitro à Ralstonia solanacearum chez six hybrides somatiques de pomme de terre (Solanum tuberosum L.) et leurs parents. Rev. Cames, Série A, Sciences et Médecine, 3 : 75-80.

Sikirou R, Assogba Komlan F, Tosso F et Agassounon T, 2011. Efficacité de la sève $\mathrm{du}$ pseudo-tronc $\mathrm{du}$ bananier contre le champignon Sclerotium rolfsii, un agent causal du flétrissement de la tomate. Bulletin de la Recherche Agronomique du Bénin, 70 : 47-55.

Sikirou R, Beed F, Ezin V, Gbehounou G, Miller SA, Wydra K, 2009. First report of bacterial wilt of tomato (Solanum lycopersicum) caused by Ralstonia solanacearum in Benin. Plant Disease, 93(5): 549.

Sikirou R, Beed F, Ezin V, Hoteigni J et Miller SA, 2017. Distribution pathological and biochemical characterization of Ralstonia solanacearum in Benin. Ann. Agri. Sci., 62 : 83-88.

Swanepoel AE, 1990. The effect of temperature on the development of wilting and on progeny tuber infection of potatoes inoculated with South African strains of biovar 2 and 3 of Pseudomonas solanacearum. Potato Res., 33 : 287-290.

Winstead NN and Kelman A, 1952. Inoculation technics for evaluating resistance to Pseudomonas solanacearum. Phytopathology, 42 : 628-634. 